\title{
3 Research Square \\ Fasting and Non-Fasting Triglycerides in Patients With Acute Ischemic Stroke
}

Jun Yup Kim

Department of Neurology, Seoul National University Bundang Hospital, Seoul National University College of Medicine

\section{Keon-Joo Lee}

Department of Neurology, Seoul National University Bundang Hospital, Seoul National University College of Medicine

Jihoon Kang

Department of Neurology, Seoul National University Bundang Hospital, Seoul National University College of Medicine

\section{Beom Joon Kim}

Department of Neurology, Seoul National University Bundang Hospital, Seoul National University College of Medicine

\section{Moon-Ku Han}

Department of Neurology, Seoul National University Bundang Hospital, Seoul National University College of Medicine

\section{Kyusik Kang}

Department of Neurology, Nowon Eulji Medical Center, Eulji University

\section{Min-Surk Kye}

Department of Neurology, Uijeonbu Eulji Medical Center, Eulji University

\section{Jong-Moo Park}

Department of Neurology, Uijeonbu Eulji Medical Center, Eulji University

\section{Tai Hwan Park}

Department of Neurology, Seoul Medical Center

\section{Hong-Kyun Park}

Department of Neurology, Inje University Ilsan Paik Hospital

\section{Yong-Jin Cho}

Department of Neurology, Inje University Ilsan Paik Hospital

\section{Keun-Sik Hong}

Department of Neurology, Inje University Ilsan Paik Hospital

\section{Kyung Bok Lee}

Department of Neurology, Soonchunhyang University Hospital, Soonchunhyang University College of Medicine 
Department of Neurology, Seoul National University Bundang Hospital, Seoul National University College of Medicine

\section{Ji Sung Lee}

Clinical Research Center, Asan Institute for Life Sciences, Asan Medical Center

\section{Juneyoung Lee}

Department of Biostatistics, Korea University College of Medicine

Hee-Joon Bae ( $\nabla$ braindoc@snu.ac.kr)

Department of Neurology, Seoul National University Bundang Hospital, Seoul National University College of Medicine

\section{Research Article}

Keywords: Triglycerides, postprandial, fasting triglycerides, non-fasting triglycerides, ischemic stroke

Posted Date: August 6th, 2021

DOl: https://doi.org/10.21203/rs.3.rs-750314/v1

License: (a) (1) This work is licensed under a Creative Commons Attribution 4.0 International License. Read Full License

Version of Record: A version of this preprint was published at Journal of Korean Medical Science on January 1st, 2022. See the published version at https://doi.org/10.3346/jkms.2022.37.e100. 


\section{Abstract}

\section{Introduction:}

Clinical implications of elevated fasting triglycerides (FTG) and non-fasting triglycerides (NFTG) in acute ischemic stroke (AIS) remain unknown. We aimed to elucidate the correlation and clinical significance of FTG and NFTG levels in AIS patients.

\section{Methods}

Using a multicenter prospective stroke registry, we identified AIS patients hospitalized within 24h of onset with available NFTG results. The primary outcome was a composite of stroke recurrence, myocardial infarction, and all-cause mortality up to one year.

\section{Results}

This study analyzed 2,176 patients. The prevalence of fasting and non-fasting hypertriglyceridemia was $11.5 \%$ and $24.6 \%$, respectively. Multivariate analysis revealed that younger age, diabetes, higher body mass index and initial systolic blood pressure were independently associated with both fasting and nonfasting hypertriglyceridemia (all $p<0.05$ ). Patients with higher quartiles of NFTG were more likely to be male, younger, ever-smokers, diabetic, and have family histories of premature coronary heart disease and stroke (all $p<0.05$ ). Similar tendencies were observed for FTG. The composite outcome was not associated with FTG or NFTG quartiles.

\section{Conclusion}

The fasting and non-fasting hypertriglyceridemia were prevalent in AIS patients and showed similar clinical characteristics and outcomes. High FTG and NFTG levels were not associated with occurrence of subsequent clinical events up to one year.

\section{Introduction}

In the general population, the lipid profile is conventionally measured in the blood collected after fasting for at least $8 \mathrm{~h} .{ }^{1}$ This is because an increase in the triglyceride (TG) level is seen during a fat tolerance test, in which patients typically consume $1 \mathrm{~g}$ fat/ $\mathrm{kg}$ of body weight. ${ }^{2,3}$ However, non-fasting TG (NFTG) has been reported to be associated with the risk of future occurrence of cardiovascular events, ${ }^{5}$ and it could be a better predictor of such events as compared to fasting TG (FTG) ${ }^{4,5}$ Furthermore, because most people eat regularly throughout the day and are strictly fasting only for a few hours in the morning, the NFTG level might be a better physiological indicator of the average TG concentrations in the blood 
rather than the FTG level. ${ }^{1,6}$ Therefore, in some countries, such as Denmark, NFTG has been the standard since 2009. ${ }^{1}$

Most of the current knowledge about the clinical significance of FTG and NFTG is from previous studies conducted on the general population. ${ }^{1,4-6}$ When discussing the role of FTG and NFTG in patients with acute ischemic stroke (AIS), the important question is whether FTG measurements are essential in the management of AIS. This study aimed to answer this question by (1) describing and comparing the FTG and NFTG concentrations in patients with AIS; (2) comparing their demographics, vascular risk factors, and stroke profiles according to the FTG and NFTG quartiles; (3) exploring the clinical determinants of the elevated FTG and NFTG levels; and (4) analyzing the impact of elevated FTG and NFTG levels on clinical outcomes.

\section{Methods}

\section{Study design and population}

This study was based on the Clinical Research Collaboration for Stroke-Korea (CRCS-K) registry, a webbased, nationwide, prospective, multicenter stroke registry established in 2008. ${ }^{7,8}$ From the registry database, we identified consecutive patients with AIS, who were admitted to the five participating hospitals within $24 \mathrm{~h}$ of symptom onset between October 2009 and November 2014, showing relevant ischemic lesions on diffusion-weighted imaging. The exclusion criteria were unavailability of information regarding the FTG or NFTG levels, unknown last mealtime or blood sampling time of lipid levels, or interval of $>8 \mathrm{~h}$ between the last mealtime and first sampling time for the random TG level (i.e., whose lipid profiles were regarded as fasting). Finally, 2,176 patients with AIS were included in this study (Supplemental Fig. 1).

The diagnostic evaluation and management of AIS in the participating centers were performed according to contemporary clinical practice guidelines, institutional protocols, and at the discretion of the treating physicians in charge of patient care. ${ }^{9}$ The collection of clinical information for monitoring and improving the quality of stroke care in the CRCS-K registry was approved by the Institutional Review Boards of the participating centers without patient consent to ensure anonymity of data and minimal risk to participants. We obtained additional approval for the use of the registry database for this study.

\section{Data collection}

Data regarding age, sex, body mass index (BMI), laboratory findings (systolic blood pressure [SBP], diastolic blood pressure, and fasting and non-fasting lipid and glucose levels), vascular risk factors (history of stroke and coronary heart disease [CHD], hypertension, diabetes mellitus, dyslipidemia, atrial fibrillation, and smoking status), family history of stroke and CHD, and stroke characteristics (stroke severity and subtypes) were obtained directly from the CRCS-K registry database. 
In all the participating centers, it was recommended to measure lipid concentrations, including total cholesterol, TG, low-density lipoprotein (LDL) cholesterol, and high-density lipoprotein (HDL) cholesterol at the time of hospitalization without intentional fasting. These were referred to as the non-fasting lipid concentrations, if the blood sampling was performed within $8 \mathrm{~h}$ from patients' last mealtime. Most blood samples for the assay of non-fasting lipid concentrations were drawn in the emergency room. Fasting lipid concentrations were measured on the first morning of hospitalization after overnight fasting.

The primary clinical outcome measure of this study was a composite of stroke recurrence, myocardial infarction, and all-cause mortality, captured prospectively up to one year after the index stroke by reviewing medical records and/or conducting structured telephonic interviews by the stroke coordinators of the participating centers. The detailed definitions and protocols for collecting clinical outcomes in the CRCS-K registry have been described elsewhere. ${ }^{7,8}$ The secondary outcome measure was stroke recurrence.

\section{Statistical analyses}

Scatter plots and Pearson correlation coefficients were used to depict and compare the distribution of fasting and non-fasting lipid levels. Baseline characteristics of the participants were compared according to FTG and NFTG quartiles using the Pearson's chi-square test for categorical variables and analysis of variance or Kruskal-Wallis tests for continuous variables.

To explore the clinical determinants of the two correlated dependent variables, fasting and non-fasting hypertriglyceridemia (defined as $\mathrm{TG} \geq 200 \mathrm{mg} / \mathrm{dL}$ ), ${ }^{10}$ we performed multivariate logistic regression analysis with the generalized estimating equation method with respect to the correlational structure between them. ${ }^{11}$ For each potential determinant, the equality of odds ratios (ORs) between fasting and non-fasting hypertriglyceridemia was examined using the Wald test.

Influences of the FTG and NFTG quartiles on clinical outcomes were evaluated using Cox proportional hazard models. Unadjusted and adjusted hazard ratios with $95 \%$ confidence intervals were estimated. Variables for adjustment were predetermined based on previous literature and clinical relevance that included age, sex, history of stroke or transient ischemic attack (TIA), history of CHD, hypertension, diabetes mellitus, hypercholesterolemia, atrial fibrillation, ever-smoking, statin use at discharge, antithrombotics at discharge, and Trial of Org 10172 in Acute Stroke Treatment (TOAST) classification with some modifications ${ }^{12}$. A two-sided $p$-value $<0.05$ was considered statistically significant. Statistical analysis was performed using SAS software version 9.4 (SAS Institute, Inc., Cary, NC, USA) and Statistical Package for the Social Sciences (IBM SPSS version 19.0.1; IBM Corporation, Armonk, NY, USA).

\section{Ethics declarations}

Ethical approval for this study was obtained from the Institutional Review Boards of the Seoul National University Bundang Hospital without patient consent to ensure anonymity of data and minimal risk to participants. The Institutional Review Boards of the Seoul National University Bundang Hospital waived the need for patient/care-giver consent. 


\section{Results}

Among 2,176 eligible patients, the distribution of fasting and non-fasting lipid concentrations was mostly bell-shaped and correlated well with each other (Supplemental Fig. 2 and Fig. 1). The mean ( \pm standard deviation) values of fasting and non-fasting lipid concentrations were 171.3 \pm 40.8 and $186.3 \pm 45.5$ $\mathrm{mg} / \mathrm{dL}$ for total cholesterol, $120.8 \pm 80.5$ and $160.1 \pm 115.3 \mathrm{mg} / \mathrm{dL}$ for TG, $43.7 \pm 12.2$ and $48.7 \pm 18.1$ $\mathrm{mg} / \mathrm{dL}$ for $\mathrm{HDL}$ cholesterol, and $103.6 \pm 34.9$ and $108.3 \pm 39.0 \mathrm{mg} / \mathrm{dL}$ for LDL cholesterol, respectively. Pearson correlation coefficients between fasting and non-fasting lipid concentrations were 0.88 for total cholesterol, 0.70 for TG, 0.60 for HDL cholesterol, and 0.85 for LDL cholesterol ( $p<0.001$ for all). Nonfasting TG concentrations tended to be higher than fasting concentrations up to $4 \mathrm{~h}$ after meals (Supplemental Fig. 3). Comparisons of non-fasting lipid concentrations among the quartiles of intervals between the last mealtime and sampling time showed a significant difference only for TG (Supplemental table 1).

When comparing the baseline characteristics according to the NFTG quartiles, patients with higher quartiles were more likely to be male, younger, and have large artery atherosclerosis with an ischemic stroke subtype and traditional vascular risk factors, including family history of premature CHD and stroke (Table 1). Similar tendencies were found in the analysis of the FTG quartiles (Supplemental table 2). 
Table 1

Baseline characteristics according to non-fasting triglyceride levels

\begin{tabular}{|c|c|c|c|c|c|}
\hline & $\begin{array}{l}<87 \\
\mathrm{mg} / \mathrm{dL} \\
(\mathrm{n}= \\
530)\end{array}$ & $\begin{array}{l}87-130 \mathrm{mg} / \mathrm{dL} \\
(\mathrm{n}=555)\end{array}$ & $\begin{array}{l}130-197 \mathrm{mg} / \mathrm{dL} \\
(\mathrm{n}=544)\end{array}$ & $\begin{array}{l}\geq 197 \\
\mathrm{mg} / \mathrm{dL} \\
(\mathrm{n}= \\
547)\end{array}$ & $\begin{array}{l}p- \\
\text { value }\end{array}$ \\
\hline Males, number (\%) & $\begin{array}{l}304 \\
(57.4)\end{array}$ & $325(58.6)$ & $339(62.3)$ & $\begin{array}{l}381 \\
(69.7)\end{array}$ & $\stackrel{<}{<.001}$ \\
\hline Age (years) & $\begin{array}{l}68.8 \pm \\
13.9\end{array}$ & $69.0 \pm 13.3$ & $66.8 \pm 12.2$ & $\begin{array}{l}63.4 \pm \\
12.4\end{array}$ & $\begin{array}{l}< \\
0.001\end{array}$ \\
\hline $\mathrm{BMI}\left(\mathrm{kg} / \mathrm{m}^{2}\right)$ & $\begin{array}{l}22.8 \pm \\
3.3\end{array}$ & $23.6 \pm 3.7$ & $23.8 \pm 3.2$ & $\begin{array}{l}24.8 \pm \\
3.1\end{array}$ & 0.08 \\
\hline $\begin{array}{l}\text { Abdominal circumference } \\
\text { (cm) }\end{array}$ & $\begin{array}{l}82.4 \pm \\
9.7\end{array}$ & $84.7 \pm 9.0$ & $85.2 \pm 9.8$ & $\begin{array}{l}87.8 \pm \\
9.2\end{array}$ & $\begin{array}{l}< \\
0.001\end{array}$ \\
\hline Initial SBP (mmHg) & $\begin{array}{l}146.5 \pm \\
26.7\end{array}$ & $152.6 \pm 27.1$ & $152.9 \pm 27.8$ & $\begin{array}{l}157.6 \pm \\
29.2\end{array}$ & $\dot{0.001}$ \\
\hline \multicolumn{6}{|l|}{ Medical history } \\
\hline Stroke or TIA, number (\%) & $\begin{array}{l}134 \\
(25.3)\end{array}$ & $133(24.0)$ & $124(22.8)$ & $\begin{array}{l}93 \\
(17.0)\end{array}$ & 0.01 \\
\hline CHD, number (\%) & $38(7.2)$ & $37(6.7)$ & $50(9.2)$ & $29(5.3)$ & 0.09 \\
\hline $\begin{array}{l}\text { Family history of premature } \\
\mathrm{CHD} \text {, number (\%) }\end{array}$ & $7(1.3)$ & $13(2.3)$ & $15(2.8)$ & $25(4.6)$ & 0.01 \\
\hline $\begin{array}{l}\text { Family history of stroke, } \\
\text { number (\%) }\end{array}$ & & & & & 0.01 \\
\hline$\geq 60$ years & $46(8.7)$ & $69(12.4)$ & $69(12.7)$ & $\begin{array}{l}78 \\
(14.3)\end{array}$ & \\
\hline$<60$ years & $14(2.6)$ & $23(4.1)$ & $18(3.3)$ & $32(5.9)$ & \\
\hline Hypertension, number (\%) & $\begin{array}{l}339 \\
(64.0)\end{array}$ & $398(71.7)$ & $389(71.5)$ & $\begin{array}{l}374 \\
(68.4)\end{array}$ & 0.02 \\
\hline Diabetes, number (\%) & $\begin{array}{l}123 \\
(23.2)\end{array}$ & $167(30.1)$ & $199(36.6)$ & $\begin{array}{l}217 \\
(39.7)\end{array}$ & $<.001$ \\
\hline
\end{tabular}

Values are expressed as mean \pm standard deviation, unless noted otherwise.

TG, triglyceride; BMI, body mass index; SBP, systolic blood pressure; TIA, transient ischemic attack; CHD, coronary heart disease; NIHSS, National Institutes of Health Stroke Scale; IQR, interquartile range; TOAST, Trial of ORG 10172 in acute stroke treatment; LAA, large artery atherosclerosis; SVO, small-vessel occlusion; HbA1c, glycated hemoglobin; FTG, fasting triglycerides; NFTG, non-fasting triglycerides; HDL, high-density lipoprotein; LDL, low-density lipoprotein.

asampling interval time was calculated as the time interval between the last meal and sampling for lipid profiles. 


\begin{tabular}{|c|c|c|c|c|c|}
\hline & $\begin{array}{l}<87 \\
\mathrm{mg} / \mathrm{dL} \\
(\mathrm{n}= \\
530)\end{array}$ & $\begin{array}{l}87-130 \mathrm{mg} / \mathrm{dL} \\
(\mathrm{n}=555)\end{array}$ & $\begin{array}{l}130-197 \mathrm{mg} / \mathrm{dL} \\
(\mathrm{n}=544)\end{array}$ & $\begin{array}{l}\geq 197 \\
\mathrm{mg} / \mathrm{dL} \\
(\mathrm{n}= \\
547)\end{array}$ & $\begin{array}{l}p- \\
\text { value }\end{array}$ \\
\hline $\begin{array}{l}\text { Hypercholesterolemia, } \\
\text { number }(\%)\end{array}$ & $\begin{array}{l}147 \\
(27.7)\end{array}$ & $172(31.0)$ & $186(34.2)$ & $\begin{array}{l}190 \\
(34.7)\end{array}$ & 0.051 \\
\hline Atrial fibrillation, number (\%) & $\begin{array}{l}191 \\
(36.0)\end{array}$ & $138(24.9)$ & $108(19.9)$ & $\begin{array}{l}83 \\
(15.2)\end{array}$ & $<0.001$ \\
\hline Ever-smoking, number (\%) & $\begin{array}{l}197 \\
(37.2)\end{array}$ & $226(40.7)$ & $247(45.4)$ & $\begin{array}{l}287 \\
(52.5)\end{array}$ & $<.001$ \\
\hline $\begin{array}{l}\text { History of statin use, number } \\
(\%)\end{array}$ & $\begin{array}{l}122 \\
(23.0)\end{array}$ & $147(26.5)$ & $114(21.0)$ & $\begin{array}{l}111 \\
(20.3)\end{array}$ & 0.06 \\
\hline \multicolumn{6}{|l|}{ Stroke profile } \\
\hline $\begin{array}{l}\text { NIHSS at admission, median } \\
\text { (IQR) }\end{array}$ & $\begin{array}{l}4(2- \\
12)\end{array}$ & $4(2-9)$ & $3(1-8)$ & $3(1-6)$ & <. \\
\hline $\begin{array}{l}\text { TOAST classification, } \\
\text { number }(\%)\end{array}$ & & & & & $\begin{array}{l}< \\
0.001\end{array}$ \\
\hline LAA & $\begin{array}{l}151 \\
(28.5)\end{array}$ & $191(34.4)$ & $203(37.3)$ & $\begin{array}{l}204 \\
(37.3)\end{array}$ & \\
\hline SVO & $\begin{array}{l}60 \\
(11.3)\end{array}$ & $89(16.0)$ & $99(18.2)$ & $\begin{array}{l}123 \\
(22.5)\end{array}$ & \\
\hline Cardioembolism & $\begin{array}{l}192 \\
(36.2)\end{array}$ & $156(28.1)$ & $105(19.3)$ & $\begin{array}{l}90 \\
(16.5)\end{array}$ & \\
\hline Other determined & $20(3.8)$ & $10(1.8)$ & $18(3.3)$ & $9(1.6)$ & \\
\hline Undetermined & $\begin{array}{l}107 \\
(20.2)\end{array}$ & $109(19.6)$ & $119(21.9)$ & $\begin{array}{l}121 \\
(22.1)\end{array}$ & \\
\hline \multicolumn{6}{|l|}{ Laboratory findings } \\
\hline HbA1c (\%) & $5.9 \pm 1.1$ & $6.2 \pm 1.2$ & $6.5 \pm 1.4$ & $6.7 \pm 1.5$ & $<.001$ \\
\hline
\end{tabular}

Values are expressed as mean \pm standard deviation, unless noted otherwise.

TG, triglyceride; BMI, body mass index; SBP, systolic blood pressure; TIA, transient ischemic attack; CHD, coronary heart disease; NIHSS, National Institutes of Health Stroke Scale; IQR, interquartile range; TOAST, Trial of ORG 10172 in acute stroke treatment; LAA, large artery atherosclerosis; SVO, small-vessel occlusion; HbA1c, glycated hemoglobin; FTG, fasting triglycerides; NFTG, non-fasting triglycerides; HDL, high-density lipoprotein; LDL, low-density lipoprotein.

aSampling interval time was calculated as the time interval between the last meal and sampling for lipid profiles. 


\begin{tabular}{|c|c|c|c|c|c|}
\hline & $\begin{array}{l}<87 \\
\mathrm{mg} / \mathrm{dL} \\
(\mathrm{n}= \\
530)\end{array}$ & $\begin{array}{l}87-130 \mathrm{mg} / \mathrm{dL} \\
(\mathrm{n}=555)\end{array}$ & $\begin{array}{l}130-197 \mathrm{mg} / \mathrm{dL} \\
(\mathrm{n}=544)\end{array}$ & $\begin{array}{l}\geq 197 \\
\mathrm{mg} / \mathrm{dL} \\
(\mathrm{n}= \\
547)\end{array}$ & $\begin{array}{l}p- \\
\text { value }\end{array}$ \\
\hline Glucose (mg/dL) & $\begin{array}{l}103.2 \pm \\
33.5\end{array}$ & $107.1 \pm 40.0$ & $113.4 \pm 58.7$ & $\begin{array}{l}122.3 \pm \\
50.7\end{array}$ & $\begin{array}{l}< \\
0.001\end{array}$ \\
\hline Total cholesterol (mg/dL) & $\begin{array}{l}156.1 \pm \\
35.9\end{array}$ & $166.1 \pm 37.6$ & $175.7 \pm 41.0$ & $\begin{array}{l}186.8 \pm \\
42.0\end{array}$ & $\begin{array}{l}< \\
0.001\end{array}$ \\
\hline FTG (mg/dL) & $\begin{array}{l}64.9 \pm \\
24.9\end{array}$ & $92.9 \pm 37.8$ & $123.2 \pm 45.5$ & $\begin{array}{l}201.0 \pm \\
106.8\end{array}$ & $\begin{array}{l}< \\
0.001\end{array}$ \\
\hline $\mathrm{HDL}(\mathrm{mg} / \mathrm{dL})$ & $\begin{array}{l}47.8 \pm \\
12.9\end{array}$ & $44.7 \pm 12.2$ & $42.7 \pm 12.1$ & $\begin{array}{l}39.9 \pm \\
10.2\end{array}$ & $<0.001$ \\
\hline LDL (mg/dL) & $\begin{array}{l}90.6 \pm \\
30.6\end{array}$ & $101.2 \pm 32.1$ & $109.6 \pm 36.2$ & $\begin{array}{l}112.9 \pm \\
36.2\end{array}$ & $\begin{array}{l}< \\
0.001\end{array}$ \\
\hline \multicolumn{6}{|l|}{ Non-fasting state } \\
\hline Glucose (mg/dL) & $\begin{array}{l}126.0 \pm \\
48.2\end{array}$ & $131.1 \pm 48.8$ & $139.5 \pm 66.7$ & $\begin{array}{l}149.2 \pm \\
70.5\end{array}$ & $\begin{array}{l}< \\
0.001\end{array}$ \\
\hline Total cholesterol (mg/dL) & $\begin{array}{l}167.3 \pm \\
39.1\end{array}$ & $180.5 \pm 41.2$ & $191.8 \pm 46.6$ & $\begin{array}{l}205.1 \pm \\
44.4\end{array}$ & $\begin{array}{l}< \\
0.001\end{array}$ \\
\hline NFTG (mg/dL) & $\begin{array}{l}63.9 \pm \\
15.5\end{array}$ & $107.1 \pm 12.6$ & $159.0 \pm 18.7$ & $\begin{array}{l}308.1 \pm \\
135.8\end{array}$ & $\begin{array}{l}< \\
0.001\end{array}$ \\
\hline $\mathrm{HDL}(\mathrm{mg} / \mathrm{dL})$ & $\begin{array}{l}52.9 \pm \\
16.5\end{array}$ & $50.0 \pm 18.2$ & $47.4 \pm 18.2$ & $\begin{array}{l}44.2 \pm \\
17.7\end{array}$ & $<0.001$ \\
\hline LDL (mg/dL) & $\begin{array}{l}96.1 \pm \\
32.7\end{array}$ & $106.3 \pm 36.3$ & $115.0 \pm 42.2$ & $\begin{array}{l}115.5 \pm \\
39.6\end{array}$ & $\begin{array}{l}< \\
0.001\end{array}$ \\
\hline Sampling interval time $(\min )^{a}$ & $\begin{array}{l}252.1 \pm \\
118.0\end{array}$ & $247.5 \pm 114.5$ & $248.3 \pm 111.1$ & $\begin{array}{l}259.4 \pm \\
107.1\end{array}$ & 0.28 \\
\hline \multicolumn{6}{|c|}{ Values are expressed as mean \pm standard deviation, unless noted otherwise. } \\
\hline \multicolumn{6}{|c|}{$\begin{array}{l}\text { TG, triglyceride; BMI, body mass index; SBP, systolic blood pressure; TIA, transient ischemic attack; } \\
\text { CHD, coronary heart disease; NIHSS, National Institutes of Health Stroke Scale; IQR, interquartile } \\
\text { range; TOAST, Trial of ORG } 10172 \text { in acute stroke treatment; LAA, large artery atherosclerosis; SVO, } \\
\text { small-vessel occlusion; HbA1c, glycated hemoglobin; FTG, fasting triglycerides; NFTG, non-fasting } \\
\text { triglycerides; HDL, high-density lipoprotein; LDL, low-density lipoprotein. }\end{array}$} \\
\hline
\end{tabular}

The prevalence of fasting and non-fasting hypertriglyceridemia ( $\geq 200 \mathrm{mg} / \mathrm{dL}$ ) was $11.5 \%(\mathrm{n}=251)$ and $24.6 \%(n=536)$, respectively. Multivariate analysis revealed that age, BMI, initial SBP, and diabetes were independently associated with both fasting and non-fasting hypertriglyceridemia; hypercholesterolemia 
and ever-smoking were associated with fasting hypertriglyceridemia; and family history of premature $\mathrm{CHD}$ and history of stroke or TIA were associated with non-fasting hypertriglyceridemia $(p<0.05)$ (Table 2). However, tests for examining the equality of ORs for each variable showed no inequality in the analyzed variables. 
Table 2

Multivariate analyses and tests for equality of odds ratios for fasting and non-fasting hypertriglyceridemia (defined as triglycerides $\geq 200 \mathrm{mg} / \mathrm{dL}$ )

\begin{tabular}{|c|c|c|c|c|c|c|c|}
\hline & \multicolumn{3}{|c|}{$\begin{array}{l}\text { Fasting } \\
\text { hypertriglyceridemia }\end{array}$} & \multicolumn{3}{|c|}{$\begin{array}{l}\text { Non-fasting } \\
\text { hypertriglyceridemia }\end{array}$} & \multirow[t]{2}{*}{$\begin{array}{l}p \text {-value for OR } \\
\text { equality }\end{array}$} \\
\hline & OR & $95 \% \mathrm{Cl}$ & $\begin{array}{l}p- \\
\text { value }\end{array}$ & OR & $95 \% \mathrm{Cl}$ & $\begin{array}{l}p- \\
\text { value }\end{array}$ & \\
\hline Male sex & 0.87 & $\begin{array}{l}0.60^{-} \\
1.25\end{array}$ & 0.44 & 1.20 & $\begin{array}{l}0.92- \\
1.57\end{array}$ & 0.19 & 0.05 \\
\hline Age, 10 years & 0.98 & $\begin{array}{l}0.97- \\
0.99\end{array}$ & 0.001 & 0.98 & $\begin{array}{l}0.98- \\
0.99\end{array}$ & 0.001 & 0.39 \\
\hline $\mathrm{BMI}, 1 \mathrm{~kg} / \mathrm{m}^{2}$ & 1.10 & $\begin{array}{l}1.06- \\
1.14\end{array}$ & $<0.001$ & 1.11 & $\begin{array}{l}1.07- \\
1.14\end{array}$ & $\begin{array}{l}< \\
0.001\end{array}$ & 0.75 \\
\hline SBP, $10 \mathrm{mmHg}$ & 1.07 & $\begin{array}{l}1.02- \\
1.13\end{array}$ & 0.003 & 1.06 & $\begin{array}{l}1.02- \\
1.11\end{array}$ & 0.003 & 0.68 \\
\hline $\begin{array}{l}\text { Family history of } \\
\text { premature CHD }\end{array}$ & 1.45 & $\begin{array}{l}0.70- \\
2.98\end{array}$ & 0.31 & 1.98 & $\begin{array}{l}1.11- \\
3.53\end{array}$ & 0.02 & 0.37 \\
\hline Family history of stroke & & & & & & & 0.37 \\
\hline None & \multicolumn{3}{|c|}{ Reference } & \multicolumn{3}{|c|}{ Reference } & \\
\hline$\geq 60$ years & 1.29 & $\begin{array}{l}0.86- \\
1.92\end{array}$ & 0.22 & 1.34 & $\begin{array}{l}0.99- \\
1.82\end{array}$ & 0.06 & \\
\hline$<60$ years & 0.88 & $\begin{array}{l}0.46- \\
1.67\end{array}$ & 0.70 & 1.34 & $\begin{array}{l}0.81- \\
2.23\end{array}$ & 0.25 & \\
\hline \multicolumn{8}{|l|}{ Medical history } \\
\hline Stroke or TIA & 0.84 & $\begin{array}{l}0.57- \\
1.22\end{array}$ & 0.35 & 0.67 & $\begin{array}{l}0.50- \\
0.90\end{array}$ & 0.01 & 0.22 \\
\hline $\mathrm{CHD}$ & 1.00 & $\begin{array}{l}0.56- \\
1.79\end{array}$ & 0.99 & 0.66 & $\begin{array}{l}0.42- \\
1.03\end{array}$ & 0.07 & 0.19 \\
\hline Hypertension & 1.10 & $\begin{array}{l}0.78- \\
1.56\end{array}$ & 0.57 & 0.96 & $\begin{array}{l}0.75- \\
1.23\end{array}$ & 0.72 & 0.36 \\
\hline Diabetes & 1.72 & $\begin{array}{l}1.28- \\
2.33\end{array}$ & 0.004 & 1.71 & $\begin{array}{l}1.37- \\
2.14\end{array}$ & $<.001$ & 0.95 \\
\hline Hypercholesterolemia & 1.55 & $\begin{array}{l}1.09- \\
2.19\end{array}$ & 0.01 & 1.24 & $\begin{array}{l}0.94- \\
1.65\end{array}$ & 0.13 & 0.20 \\
\hline Atrial fibrillation & 0.98 & $\begin{array}{l}0.52- \\
1.85\end{array}$ & 0.96 & 0.75 & $\begin{array}{l}0.51- \\
1.11\end{array}$ & 0.16 & 0.36 \\
\hline
\end{tabular}

OR, odds ratio; $\mathrm{BMI}$, body mass index; SBP, systolic blood pressure; CHD, coronary heart disease; TIA, transient ischemic attack. 


\begin{tabular}{|c|c|c|c|c|c|c|c|}
\hline & \multicolumn{3}{|c|}{$\begin{array}{l}\text { Fasting } \\
\text { hypertriglyceridemia }\end{array}$} & \multicolumn{3}{|c|}{$\begin{array}{l}\text { Non-fasting } \\
\text { hypertriglyceridemia }\end{array}$} & \multirow[t]{2}{*}{$\begin{array}{l}p \text {-value for OR } \\
\text { equality }\end{array}$} \\
\hline & OR & $95 \% \mathrm{Cl}$ & $\begin{array}{l}p- \\
\text { value }\end{array}$ & OR & $95 \% \mathrm{Cl}$ & $\begin{array}{l}p- \\
\text { value }\end{array}$ & \\
\hline Ever-smoking & 1.43 & $\begin{array}{l}1.02- \\
2.02\end{array}$ & 0.04 & 1.15 & $\begin{array}{l}0.90- \\
1.48\end{array}$ & 0.27 & 0.18 \\
\hline History of statin use & 0.66 & $\begin{array}{l}0.42- \\
1.01\end{array}$ & 0.06 & 0.75 & $\begin{array}{l}0.54- \\
1.06\end{array}$ & 0.10 & 0.49 \\
\hline $\begin{array}{l}\text { Sampling interval time } \\
\text { (min) }\end{array}$ & 1.02 & $\begin{array}{l}0.95- \\
1.10\end{array}$ & 0.51 & 1.04 & $\begin{array}{l}0.98- \\
1.10\end{array}$ & 0.19 & 0.71 \\
\hline
\end{tabular}

Compared to the lowest quartile of NFTG, the highest quartile was negatively associated with the composite outcome in the unadjusted analysis, but this association was not statistically significant after adjustments for age and sex (Fig. 2, Supplemental table 3). The FTG and NFTG quartiles were not associated with any outcome variables after adjustments for predetermined potential confounders (Fig. 2, Supplemental tables 3 and 4). Moreover, fasting and non-fasting hypertriglyceridemia were not associated with an increased risk of any clinical outcomes (Table 3 ).

Table 3

Hazard ratios (HR) according to fasting and non-fasting hypertriglyceridemia ${ }^{a}$

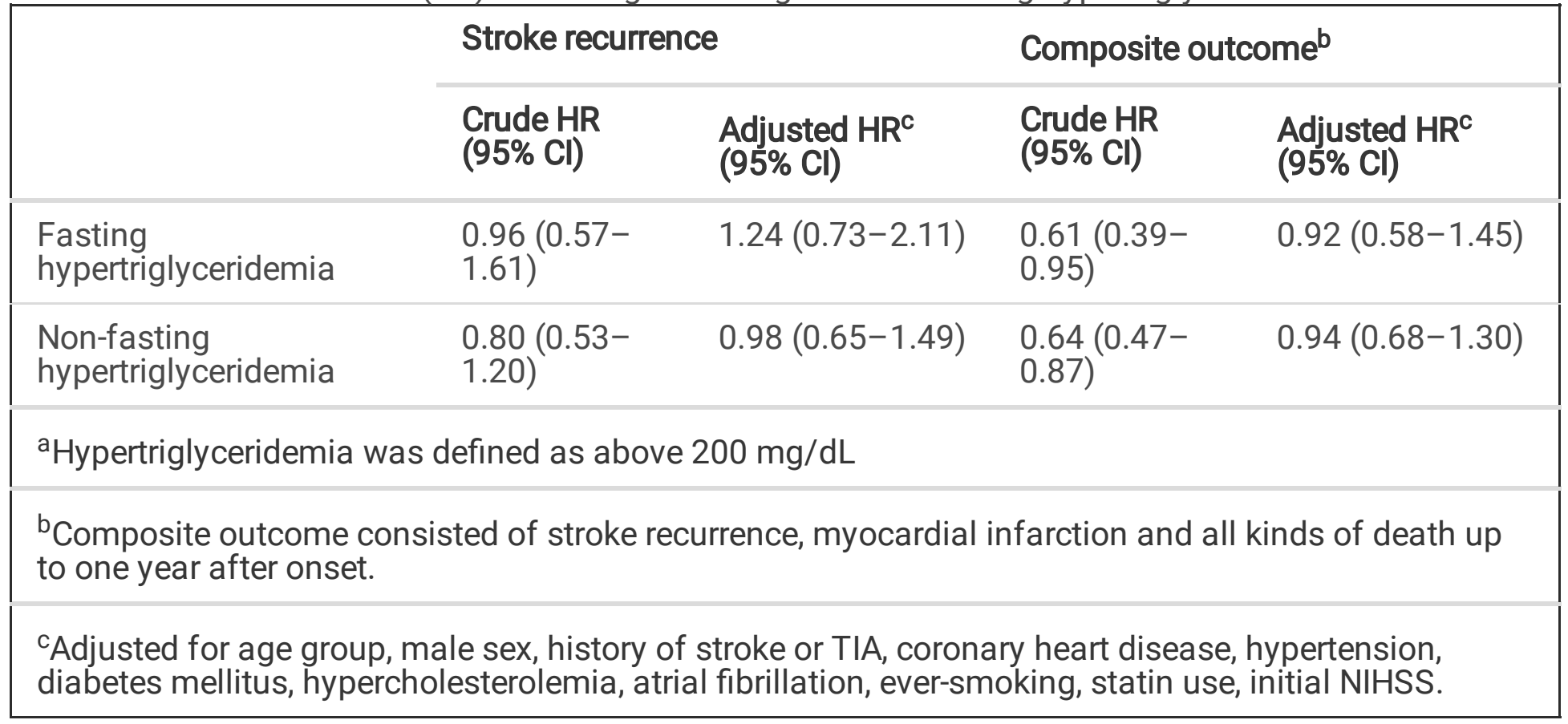

\section{Discussion}


This study found that (1) fasting and non-fasting lipid concentrations were well correlated; (2) fasting and non-fasting hypertriglyceridemia were prevalent in AIS patients; (3) fasting and non-fasting hypertriglyceridemia were associated with younger age, diabetes, and higher BMI and initial SBP; and (4) FTG and NFTG levels were not associated with the clinical outcomes.

Following food ingestion, TG is transported from the small intestines by chylomicrons through the bloodstream. Lipolysis of TG within chylomicrons, catalyzed by lipoprotein lipase, transforms these particles into atherogenic TG-remnant lipoproteins. Elevated postprandial TG levels, reflecting either higher peak levels or delay in clearance of TG-rich particles, can lead to accumulation of these atherogenic particles. ${ }^{13}$ The data presented in Supplement Table 1 corresponds to the expected time course of postprandial TG metabolism. In response to food intake, TG concentrations typically reach their peaks approximately $4 \mathrm{~h}$ after meals and decline thereafter. ${ }^{14}$ Thus, postprandial TG levels are more physiologic and better associated with thrombogenic conditions, such as ischemic stroke. Furthermore, our study suggests that patients with elevated FTG and NFTG levels show similar characteristics. Therefore, NFTG can replace FTG in the diagnostic evaluation of patients with AIS.

Several biological mechanisms provide plausible explanations about the associations between high FTG and NFTG concentrations and young age, higher BMI, higher SBP, and diabetes, which are components of the metabolic syndrome. Hypertriglyceridemia is a feature of dyslipidemia seen in type 2 diabetes and metabolic syndrome, which may also include insulin resistance, abdominal obesity, and hypertension. ${ }^{10}$ Hyperinsulinemia leads to an increase in the production of very-low-density lipoprotein. ${ }^{15}$ Hyperglycemia also impairs removal of TG-rich lipoproteins from circulation. Patients with poorly controlled diabetes have higher TG levels than those with well-controlled diabetes. Postprandial hyperlipidemia in diabetic patients appears to be prolonged, indicating that the arteries are exposed to atherogenic particles for extended periods. ${ }^{15,16}$

In this study, both fasting and non-fasting TG levels were not associated with the composite outcome comprising of stroke recurrence, myocardial infarction, or all-cause mortality up to one year after the index stroke. Contradicting our results, in patients with established cardiovascular disease or risk factors, higher TG levels were reportedly associated with an increased risk of recurrent vascular events ${ }^{17}$, and reducing TG levels lowered the risk of ischemic events ${ }^{18}$. There are some explanations for these discrepancies. First, it has been reported that high TG levels might be associated with milder stroke severity and better outcomes in patients with AIS. ${ }^{19-21}$ In our study, NFTG negatively correlated with the initial National Institutes of Health Stroke Scale scores (Pearson correlation coefficient $=-1.33, p<$ 0.001). Second, TG could paradoxically prevent lipotoxicity, and subsequently poor outcome. ${ }^{22,23}$ Third, treatment with statin at discharge might attenuate the effect of high TG on outcome. The proportion of patients treated with statin at discharge was $88.5 \%$ in our study. In a previous study that reported a significant correlation between TG and recurrent stroke, the statin prescription rate was $36 \% .{ }^{24}$ 
Our study had several limitations. First, it only included Korean patients, and these results may not be generalizable to other populations. Second, the proportion of patients excluded from the analysis was $63 \%$, accounting for a fairly large proportion. Particularly, when compared to patients excluded from this

study, the proportion of young males, BMI, and prevalence of hyperlipidemia was relatively higher among the included patients, but the prevalence of diabetes did not differ between them (Supplemental table 5). The possibility of selection bias cannot be excluded, but the differences seemed considerably small to affect the clinical outcomes. Third, we did not have information on other possible factors affecting the lipid levels, such as foods, drugs, and nutritional state.

\section{Conclusion}

In conclusion, our study shows that AIS patients with fasting and non-fasting hypertriglyceridemia may not differ in their clinical characteristics and outcomes. Furthermore, fasting and non-fasting TG levels were not associated with subsequent clinical events, including stroke recurrence, myocardial infarction, and all-cause mortality up to one year.

\section{Declarations}

\section{Acknowledgements}

None

\section{Author contributions}

J.Y. Kim: Participated in literature search, study design, data collection, analysis, interpretation, and writing the manuscript which was then developed further in collaboration with the other authors. M-S Kye, K-J Lee, J. Kang, B.J. Kim, M-K Han, K. Kang, J-M Park, T.H. Park, H-K Park, Y-J Cho, K-S Hong, K. B. Lee, M.S. Jang: Participated in the study design, data collection, and revision of the manuscript. J.S. Lee, J. Lee: Primarily active in statistical methodology and data interpretation, but also in manuscript writing in collaboration. H-J Bae: Participated in study design, data collection and interpretation. Revised the manuscript and approved the final version. All authors gave final approval of the manuscript.

\section{Competing interests}

$\mathrm{H}-\mathrm{J}$ Bae is the principal investigator. He is also a member of the steering committee, and/or a site investigator of multicenter clinical trials or clinical studies that are sponsored by BMS Korea, Shinpoong Pharm. Co. Ltd., Bayer, Boehringer Ingelheim, Daiichi-Sankyo, Esai, AstraZeneca Korea, Servier Korea, Yuhan Corporation, Jeil Pharmaceutical Co., Shire Korea, JLK inspection, Chong Gun Dang Pharmaceutical Corp., and Dong-A Pharmaceutical. He has received lecture honoraria from Esai, Shire Korea, Amgen, and Otsuka Korea outside of this work. The other authors declare no competing interests. 
This research was partially supported by the research fund of the Korea Centers for Disease Control and Prevention (2020ER620200\#) and Daewoong Pharmaceutical Co. Ltd. The funding sources had no role in the study design, data collection, analysis and interpretation, as well as preparation, review, and approval of the manuscript.

\section{References}

1. Nordestgaard, B. G. \& Varbo, A. Triglycerides and cardiovascular disease., 384, 626-635 https://doi.org/10.1016/S0140-6736(14)61177-6 (2014).

2. Cohn, J. S., McNamara, J. R., Cohn, S. D., Ordovas, J. M. \& Schaefer, E. J. Postprandial plasma lipoprotein changes in human subjects of different ages. J Lipid Res, 29, 469-479 (1988).

3. Schaefer, E. J. et al. Comparison of fasting and postprandial plasma lipoproteins in subjects with and without coronary heart disease. Am J Cardiol, 88, 1129-1133 (2001).

4. Bansal, S. et al. Fasting compared with nonfasting triglycerides and risk of cardiovascular events in women. JAMA, 298, 309-316 https://doi.org/10.1001/jama.298.3.309 (2007).

5. Nordestgaard, B. G., Benn, M., Schnohr, P. \& Tybjaerg-Hansen, A. Nonfasting triglycerides and risk of myocardial infarction, ischemic heart disease, and death in men and women. JAMA, 298, 299-308 https://doi.org/10.1001/jama.298.3.299 (2007).

6. Langsted, A., Freiberg, J. J. \& Nordestgaard, B. G. Fasting and nonfasting lipid levels: influence of normal food intake on lipids, lipoproteins, apolipoproteins, and cardiovascular risk prediction., 118, 2047-2056 https://doi.org/10.1161/CIRCULATIONAHA.108.804146 (2008).

7. Kim, B. J. et al. Current status of acute stroke management in Korea: a report on a multicenter, comprehensive acute stroke registry. Int J Stroke, 9, 514-518 https://doi.org/10.1111/ijs.12199 (2014).

8. Kim, B. J. et al. Case characteristics, hyperacute treatment, and outcome information from the clinical research center for stroke-fifth division registry in South Korea. J Stroke, 17, 38-53 https://doi.org/10.5853/jos.2015.17.1.38 (2015).

9. Adams, H. P. Jr. et al. Guidelines for the early management of adults with ischemic stroke: a guideline from the American Heart Association/American Stroke Association Stroke Council, Clinical Cardiology Council, Cardiovascular Radiology and Intervention Council, and : The American Academy of Neurology affirms the value of this guideline as an educational tool for neurologists. Circulation 115, e478-534, doi:10.1161/CIRCULATIONAHA.107.181486 (2007).

10. Expert Panel on Detection. E. \& Treatment of High Blood Cholesterol in, A. Executive Summary of The Third Report of The National Cholesterol Education Program (NCEP) Expert Panel on Detection, Evaluation, And Treatment of High Blood Cholesterol In Adults (Adult Treatment Panel III). JAMA, 285, 2486-2497 (2001).

11. Gauvreau, K. \& Pagano, M. The Analysis of Correlated Binary Outcomes Using Multivariate Logistic Regression. Biom. J, 39, 309-325 https://doi.org/10.1002/bimj.4710390306 (1997). 
12. Ko, Y. et al. MRI-based Algorithm for Acute Ischemic Stroke Subtype Classification. J Stroke, 16, 161172 https://doi.org/10.5853/jos.2014.16.3.161 (2014).

13. Ginsberg, H. N. New perspectives on atherogenesis: role of abnormal triglyceride-rich lipoprotein metabolism., 106, 2137-2142 (2002).

14. Boquist, S. et al. Alimentary lipemia, postprandial triglyceride-rich lipoproteins, and common carotid intima-media thickness in healthy, middle-aged men., 100, 723-728 (1999).

15. Coughlan, B. J. \& Sorrentino, M. J. Does hypertriglyceridemia increase risk for CAD? Growing evidence suggests it plays a role. Postgrad Med, 108, 77-84 https://doi.org/10.3810/pgm.2000.12.1314 (2000).

16. Kreisberg, R. A. Diabetic dyslipidemia.Am J Cardio/82, 67U-73U; discussion 85U-86U (1998).

17. van de Woestijne, A. P. et al. Plasma triglyceride levels increase the risk for recurrent vascular events independent of LDL-cholesterol or nonHDL-cholesterol. Int J Cardiol, 167, 403-408 https://doi.org/10.1016/j.jijcard.2012.01.008 (2013).

18. Bhatt, D. L. et al. Cardiovascular Risk Reduction with Icosapent Ethyl for Hypertriglyceridemia. N Engl J Med, 380, 11-22 https://doi.org/10.1056/NEJMoa1812792 (2019).

19. Pikija, S. et al. Higher serum triglyceride level in patients with acute ischemic stroke is associated with lower infarct volume on CT brain scans. Eur Neurol, 55, 89-92 https://doi.org/10.1159/000092780 (2006).

20. Weir, C. J., Sattar, N., Walters, M. R. \& Lees, K. R. Low triglyceride, not low cholesterol concentration, independently predicts poor outcome following acute stroke. Cerebrovasc Dis, 16, 76-82 https://doi.org/10.1159/000070119 (2003).

21. Dziedzic, T., Slowik, A., Gryz, E. A. \& Szczudlik, A. Lower serum triglyceride level is associated with increased stroke severity., 35, e151-152 https://doi.org/10.1161/01.STR.0000128705.63891.67 (2004).

22. Eun, M. Y. et al. Age-dependent predictors for recurrent stroke: the paradoxical role of triglycerides. Eur Neurol, 69, 171-178 https://doi.org/10.1159/000345480 (2013).

23. Listenberger, L. L. et al. Triglyceride accumulation protects against fatty acid-induced lipotoxicity. Proc Natl Acad Sci U S A, 100, 3077-3082 https://doi.org/10.1073/pnas.0630588100 (2003).

24. Zhao, L. et al. Association between atherogenic dyslipidemia and recurrent stroke risk in patients with different subtypes of ischemic stroke. International Journal of Stroke, 10, 752-758 (2015).

\section{Figures}



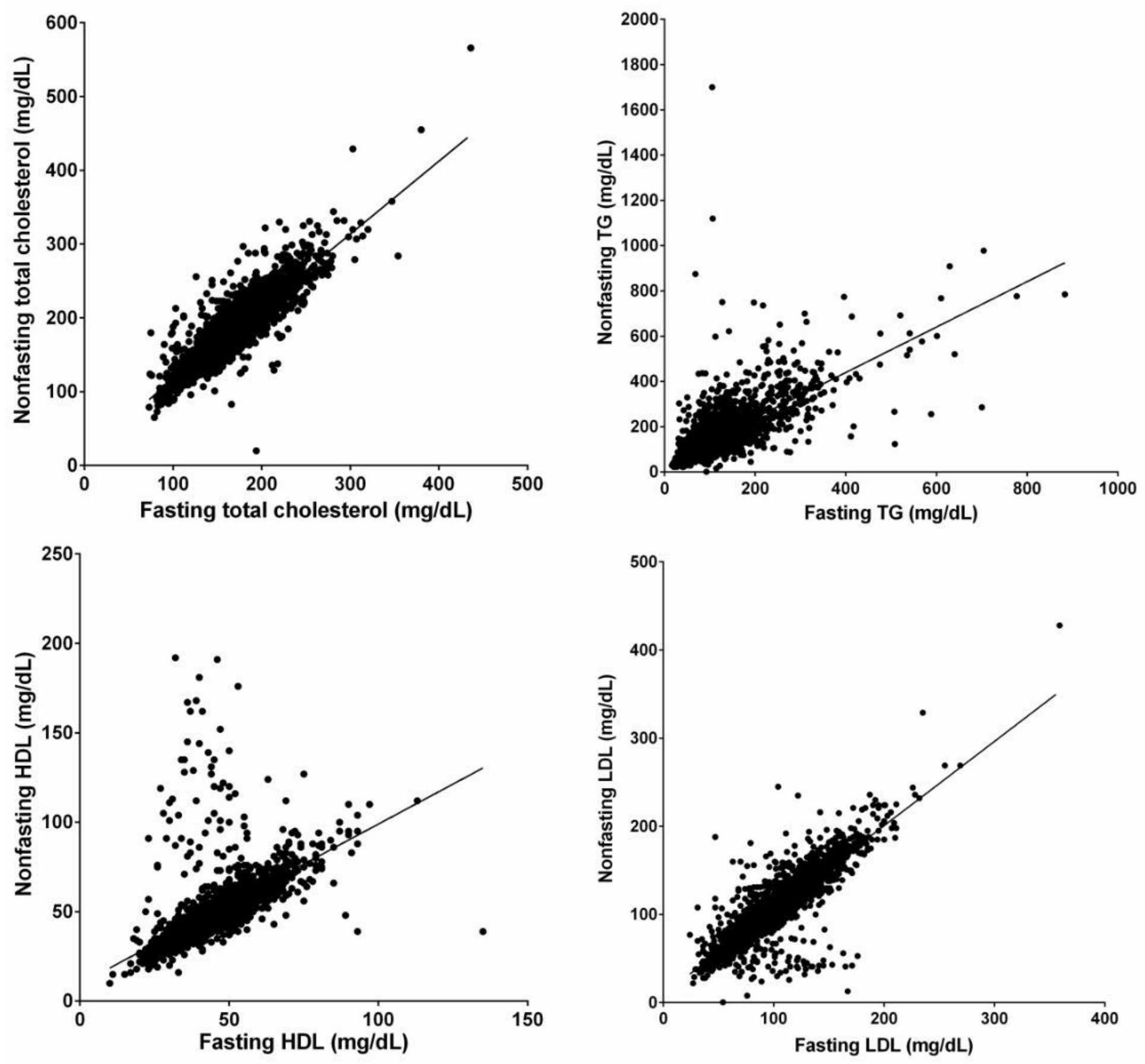

Figure 1

Correlation analyses between fasting and non-fasting lipid profiles Fasting and non-fasting lipid profiles were well correlated for total cholesterol, TG, HDL, and LDL. Pearson's correlation coefficients were 0.88 for total cholesterol, 0.70 for TG, 0.60 for $\mathrm{HDL}$, and 0.85 for $\mathrm{LDL}$ ( $\mathrm{p}<0.001$ for all). (TG, triglycerides; HDL, high-density lipoprotein; LDL, low-density lipoprotein) 


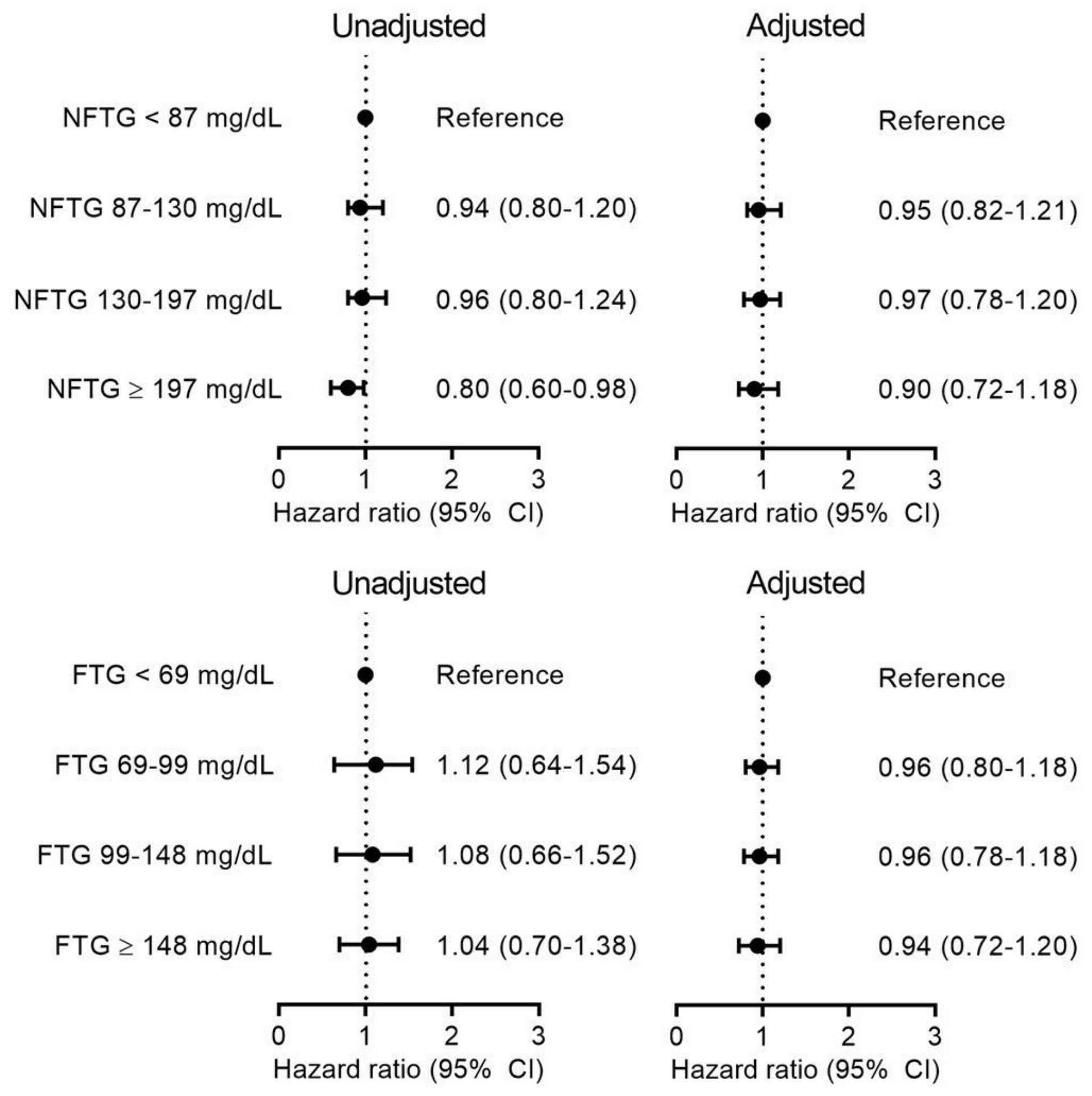

Figure 2

Cox's proportional hazard model according to the quartiles of fasting and non-fasting triglycerides for the composite outcome Hazard ratios were adjusted for age, sex, history of stroke or transient ischemic attack, history of coronary heart disease, hypertension, diabetes mellitus, atrial fibrillation, ever-smoking, history of statin use, and Trial of Org 10172 in acute stroke treatment classification. The composite outcome consisted of stroke recurrence, myocardial infarction, and all-cause mortality up to one year after the index stroke. (Cl, confidence interval; NFTG, non-fasting triglycerides; FTG, fasting triglycerides) 


\section{Supplementary Files}

This is a list of supplementary files associated with this preprint. Click to download.

- Supplementalmaterials.docx 